\title{
Correction to: "Meme Wars": A Brief Overview of Memetics and Some Essential Context
}

\author{
Michael P. Schlaile
}

\section{Correction to:}

Chapter 2 in: M. P. Schlaile (ed.), Memetics and Evolutionary Economics, Economic Complexity and Evolution, https://doi.org/10.1007/978-3-030-59955-3_2

In the original version of the book, In Chapter 2, the word "muchlonger" in page 15 is now corrected as "much longer" and the word "several doctoraldissertations" in page 17 is now corrected as "several doctoral dissertations". The chapter and book have been updated with the change. 